\title{
Inhibition of tumor angiogenesis by oral etoposide
}

\author{
DIPAK PANIGRAHY ${ }^{1,2}$, ARJA KAIPAINEN ${ }^{3}$, CATHERINE E. BUTTERFIELD ${ }^{1}$, DEVINEY M. CHAPONIS ${ }^{1,2}$, \\ ANDREA M. LAFORME ${ }^{1,2}$, JUDAH FOLKMAN $^{1}$ and MARK W. KIERAN ${ }^{1,2}$
}

\author{
${ }^{1}$ Vascular Biology Program, Children's Hospital Boston, Department of Surgery, Harvard Medical School; \\ ${ }^{2}$ Division of Pediatric Hematology/Oncology, Dana-Farber Cancer Institute, Harvard Medical School, Boston, MA, USA; \\ ${ }^{3}$ Department of Biochemistry and Molecular Biology, University of Calgary, Calgary, Canada
}

Received May 19, 2010; Accepted June 4, 2010

DOI: $10.3892 / \mathrm{etm} .2010 .127$

\begin{abstract}
The chemotherapeutic agent etoposide is a topoisomerase II inhibitor widely used for cancer therapy. Low-dose oral etoposide, administered at close regular intervals, has potent anti-tumor activity in patients who are refractory to intravenous etoposide; however, the mechanism remains unclear. Since endothelial cells may be more sensitive than tumor cells to chemotherapy agents, we determined the effects of etoposide alone and in combination with oral cyclooxygenase- 2 inhibitors and peroxisome-proliferator activated receptor $\gamma$ ligands on angiogenesis and tumor growth in xenograft tumor models. Optimal anti-angiogenic (metronomic) and anti-tumor doses of etoposide on angiogenesis, primary tumor growth and metastasis were established alone and in combination therapy. Etoposide inhibited endothelial and tumor cell proliferation, decreased vascular endothelial growth factor (VEGF) production by tumor cells and suppressed endothelial tube formation at non-cytotoxic concentrations. In our in vivo studies, oral etoposide inhibited fibroblast growth factor 2 and VEGF-induced corneal neovascularization, VEGF-induced vascular permeability and increased levels of the endogenous angiogenesis inhibitor endostatin in mice. In addition, etoposide inhibited Lewis lung carcinoma (LLC) and human glioblastoma (U87) primary tumor growth as well as spontaneous lung metastasis in a LLC resection model. Furthermore, etoposide had synergistic anti-tumor activity in combination with celecoxib and rosiglitazone, which are also oral anti-angiogenic and anti-tumor agents. Etoposide inhibits angiogenesis in vitro and in vivo by indirect and direct mechanisms of action. Combining etoposide with celecoxib and rosiglitazone increases its efficacy and merits further investigation in future clinical trials to determine the potential usefulness of etoposide in combinatory anti-angiogenic chemotherapy.
\end{abstract}

Correspondence to: Dr Mark Kieran, Division of Pediatric Oncology, Dana-Farber Cancer Institute, Harvard Medical School, Boston, MA 02115, USA

E-mail: mark_kieran@dfci.harvard.edu

Key words: etoposide, tumor angiogenesis, metronomic chemotherapy, oral combination therapy, peroxisome-proliferator activated receptor

\section{Introduction}

Daily administered, low-dose, cytotoxic, chemotherapeutic drugs were initially shown by Browder et al to preferentially target the endothelium of the tumor vasculature (1). When cyclophosphamide was administered in low frequent doses, as opposed to the maximally tolerated dose every three weeks, potent tumor suppression was achieved as a result of endothelial cell apoptosis. This anti-angiogenic, or metronomic, chemotherapeutic approach avoids the development of tumor cell resistance by targeting the proliferating endothelial cells required for tumor neovascularization (2-4). Furthermore, the greater sensitivity of endothelial cells in comparison to tumor cells allows for significantly lower doses of the drug to be effective, thus improving tolerability $(5,6)$. Anti-angiogenic chemotherapy has entered clinical trials for various vascular tumors refractory to conventional chemotherapy (4,7-9). In our study, $40 \%$ of children with recurrent or progressive cancer, treated with daily low-dose oral etoposide alternating every 21 days with daily low-dose oral cyclophosphamide combined with daily oral thalidomide and celecoxib, exhibited a prolonged or persistent progression-free disease status (7).

Etoposide (VP16), a topoisomerase II inhibitor, is a semisynthetic derivative of podophyllotoxin introduced in cancer clinical trials in 1971 and FDA-approved in 1983. It is an alkaloid cytotoxic drug that binds to and inhibits topoisomerase II-DNA function in ligating cleaved DNA molecules, resulting in the accumulation of single- or doublestrand DNA breaks and stops the cell cycle at the late $\mathrm{S}$ and G2 phases (10). Daily oral etoposide is effective for the treatment of several tumors, including non-small cell lung cancer, recurrent medulloblastoma and neuroblastoma, after these tumors have developed resistance to the maximally tolerated doses of intravenous etoposide $(11,12)$. Additionally, platinumresistant epithelial ovarian cancer, metastatic breast cancer and pediatric recurrent sarcomas have been successfully treated with oral etoposide (13-15). When compared to intravenous administration, treatment with oral etoposide increased the response rate in patients with small-cell lung and advanced breast cancers $(16,17)$. However, the mechanism by which low-dose oral etoposide inhibits the growth of tumors resistant to maximally tolerated higher-dose intravenous etoposide has not been extensively studied. 
We hypothesize that tumor endothelium is a potential target of low-dose oral etoposide, since the primary tumor and metastatic growth are dependent on angiogenesis (18). This hypothesis is supported by observations that etoposide inhibits the proliferation of endothelial cells (19). In fact, endothelial cells were found to be more sensitive to etoposide than tumor cells in vitro (20), suggesting that the anti-tumor effect of etoposide may, in part, be mediated through the endothelium. Therefore, we investigated the role of etoposide in tumor angiogenesis. We report that etoposide inhibits primary tumor growth and metastasis through anti-angiogenic and direct antitumor effects. Oral administration of etoposide allows it to be easily incorporated into chemotherapy regimens and supports its addition to the growing class of oral anti-angiogenic drugs for cancer therapy.

\section{Materials and methods}

Cells and reagents. Bovine capillary endothelial (BCE) cells were maintained on gelatinized plastic in Dulbecco's modified Eagle's medium (DMEM) low glucose $+10 \%$ bovine calf serum. Human umbilical vein endothelial cells (HUVECs) were maintained in EBM-2 media. Lewis lung carcinoma (LLC), fibrosarcoma (T241), glioblastoma (U87), breast (MDA-MB 231) and K1000 [a tumor cell line that expresses and secretes high levels of fibroblast growth factor 2 (FGF2)] cells were cultured in DMEM $+10 \%$ heat-inactivated FBS + $1 \%$ penicillin streptomycin glutamine. For in vitro studies, etoposide (VP-16) (Sigma, St. Louis, MO, USA) was used and for in vivo studies, clinical grade IV solution was utilized.

Vascular endothelial growth factor (VEGF) ELISA. Tumor cells that were known to secrete high levels of VEGF (U87 glioblastoma and LLC) were plated at $15 \times 10^{3}$ cells per well (6-well plates), and $24 \mathrm{~h}$ later were treated with etoposide or vehicle. Medium containing the drugs was changed on Days 3 and 5. On Day 6, the medium was collected, and VEGF was assayed by ELISA (R\&D Systems Inc., Minneapolis, MN, USA).

Angiogenesis assays. Endothelial cell proliferation was assayed as described (21) at $15 \times 10^{3}$ cells per well. For tumor cell proliferation, cells were plated at $5 \times 10^{3}$ cells per well. Endothelial cell tubes were formed by combining HUVECs $\left(5 \times 10^{4}\right.$ cells/well) with varying concentrations of etoposide or vehicle on Matrigel- (Collaborative Biochemical, Bedford, MA, USA) coated 24-well plates. The animal experiments were performed in accordance with IRB-approved protocols at Children's Hospital Boston.

For the corneal neovascularization assay, $80 \mathrm{ng}$ FGF2 or $160 \mathrm{ng}$ VEGF pellets were implanted into C57BL/6 mice (Jackson Labs, Bar Harbor, ME, USA) (22). Etoposide was administered daily over 6 days by gavage in $0.5 \%$ methylcellulose, and control mice received vehicle $(0.5 \%$ methylcellulose).

For tumor studies, LLC was injected subcutaneously as described (21). Glioblastoma (U87) and T241 fibrosarcoma were injected subcutaneously $\left(1 \times 10^{6}\right.$ cells in $\left.0.1 \mathrm{ml} \mathrm{PBS}\right)$ into 6-week-old male severe combined immunodeficient $(\mathrm{MGH}$, Boston, MA, USA) or C57BL/6 mice, respectively. Once tumors were $100-150 \mathrm{~mm}^{3}$, mice were randomized into treatment and vehicle groups. Etoposide, celecoxib, rosiglitazone and/or cyclophosphamide were administered by daily gavage for 14-40 days. Tumors were measured every 3-7 days, and the volume was calculated as width ${ }^{2} \mathrm{x}$ length $\mathrm{x} 0.52$.

For metastasis studies, LLC tumors were resected 15 days after implantation as described (21). After LLC resection, mice were treated with etoposide or vehicle for 16 days when control mice became terminally ill. On the last day of treatment, the statistical difference between the treatment and control groups was determined by the Student's t-test. A p-value $<0.05$ was accepted as significant.

Miles vascular permeability assay. One to two days prior to the experiment, mice were shaved to expose the skin. Mice were anesthetized with intraperitoneally injected Avertin and injected with $1 \%$ Evan's blue dye, either by tail vein or through the orbital plexus. VEGF (50 $\mu \mathrm{l}$ of $1 \mathrm{ng} / \mu \mathrm{l})$ and $50 \mu \mathrm{l}$ of saline or PBS with $0.05 \%$ gelatin were injected intradermally using a 30-gauge needle into the skin overlying the back. Similar experiments were performed by injecting $5 \mu \mathrm{l}$ of VEGF, saline or PBS intra-dermally into the ears. After $10 \mathrm{~min}$, the animals were euthanized, and the skin was opened and exposed to assess the intensity of Evan's blue dye extravasations. The areas of blue skin (vascular leak) were removed and placed into formamide for 5 days. The intensity of the reaction was quantified by reading the samples at a wavelength of $620 \mathrm{~nm}$ on a SpectraMax plate reader.

Immunohistochemistry. For PECAM1, the sections of tumors were treated with $40 \mu \mathrm{g} / \mathrm{ml}$ proteinase $\mathrm{K}$ (Roche Diagnostics Corp.) for $25 \mathrm{~min}$ at $37^{\circ} \mathrm{C}$. PECAM1 was amplified using tyramide signal amplification direct and indirect kits (PerkinElmer Life Sciences, Boston, MA, USA). For computer-enhanced imaging of tumors, histological sections were analyzed for vessel density by computerized densitometric imaging (Corel Photo Paint and IP Lab software). The degree of vascularization was quantified over the entire tumor section and expressed as a ratio of vessel area (PECAM1) to tumor area. Total fields scored per tumor were 67-70. For control and etoposide-treated tumors, 4 animals/group were evaluated.

\section{Results}

Etoposide has direct and indirect anti-angiogenic and antitumor activity in vitro

Direct effects. To investigate the effects of etoposide on endothelial cell proliferation, we stimulated the proliferation of BCE cells with FGF2, a potent mitogen for BCE cells, in a standard proliferation assay. Etoposide inhibited FGF2induced proliferation of BCE cells in a dose-dependent manner, with a maximal inhibition of $80 \%$ after a 72 -h incubation period at $2.5 \mu \mathrm{M}$, a concentration easily achieved orally in humans (Fig. 1A). Similarly, etoposide inhibited VEGFinduced proliferation of HUVECs up to $80 \%$ at $2.5 \mu \mathrm{M}$ (Fig. 1B). We next determined whether etoposide inhibits tumor cells at similar doses as those applied to endothelial cells. Etoposide inhibited the proliferation of human tumor cells, including glioblastoma (U87) and breast (MDA-MB 231), 
A
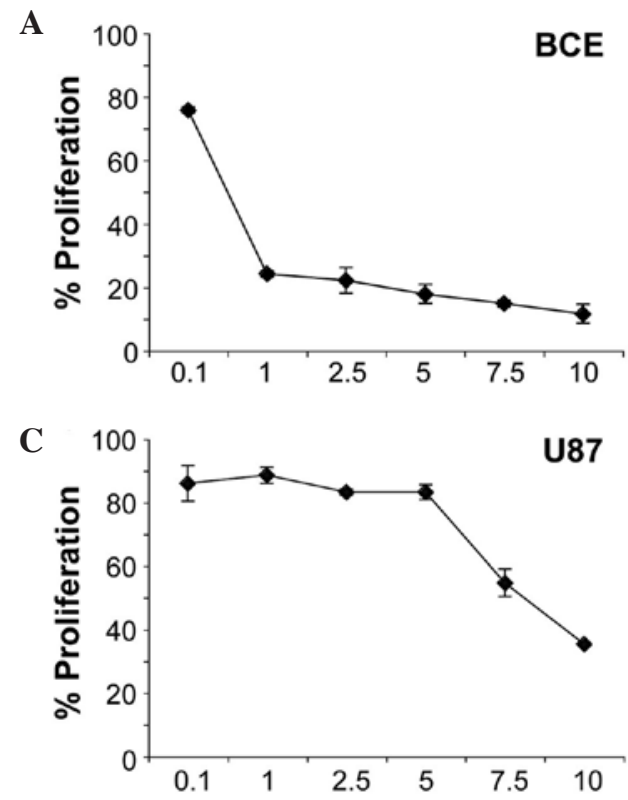

$\mathbf{E}$

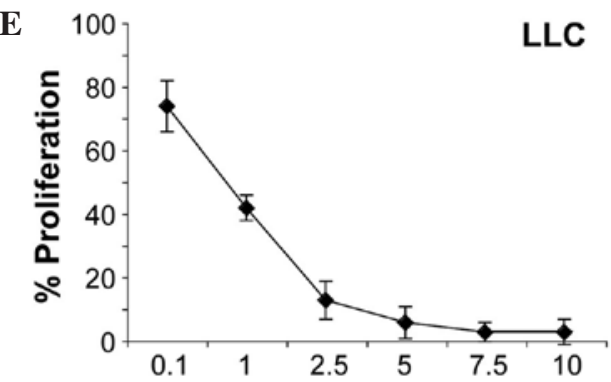

G

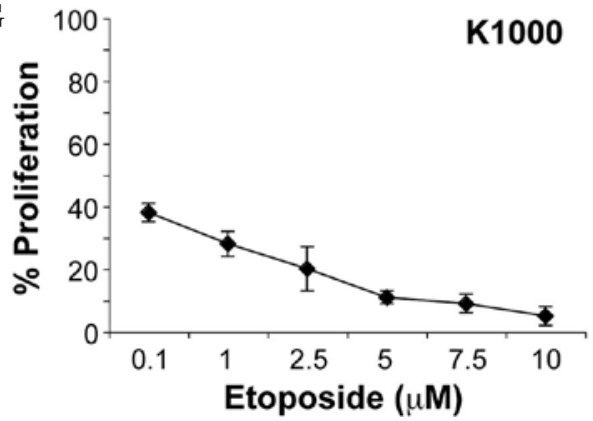

B

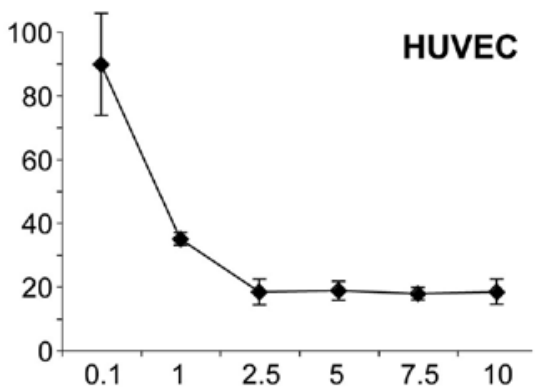

D

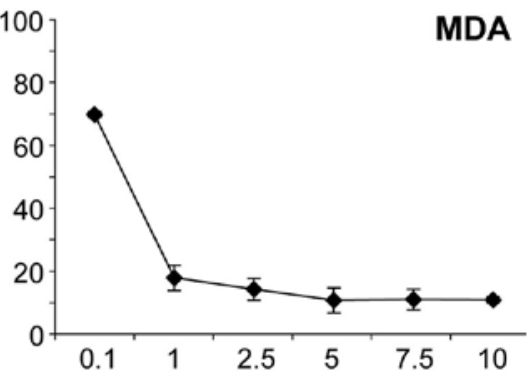

F

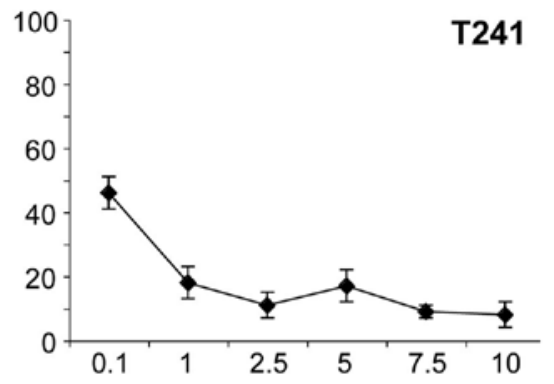

H

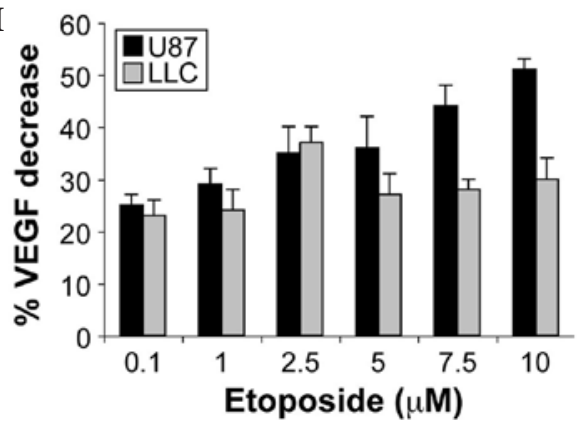

Figure 1. Etoposide has anti-angiogenic and anti-tumor cell effects in vitro. (A) The proliferation percentage of BCE cells was determined by comparing cells exposed to an angiogenic stimulus (FGF2) alone with those exposed to FGF2 and etoposide, relative to unstimulated cells (5\% calf serum). (B) The proliferation percentage of HUVECs was determined by comparing cells exposed to an angiogenic stimulus (VEGF) alone with those exposed to VEGF and etoposide. (C) The proliferation percentage of tumor cells (glioblastoma U87) was determined by comparing cells grown in media $+10 \%$ FBS and etoposide to starved cells $(0.5 \%$ FBS). The effect of etoposide on the proliferation of (D) breast carcinoma cells (MDA-MB 231), (E) LLC, (F) T241 fibrosarcoma and (G) K1000 cells. (H) VEGF levels (expressed as decreased percentage) in U87 and LLC cells 6 days after treatment with etoposide.

differentially because of the primary resistance of these cell lines (Fig. 1C and D). Murine tumor cell lines, LLC and T241 fibrosarcoma demonstrated sensitivity to etoposide (Fig. 1E-G).

Indirect effects. To determine whether etoposide inhibits angiogenesis by down-regulating tumor-secreted growth factors, we measured VEGF levels in tumor-conditioned media via ELISA. The tumor cell lines glioblastoma (U87 resistant to etoposide) and LLC (sensitive to etoposide) secreted substantial amounts of VEGF: 20,000 and 938 pg/10 6 cells, respectively. Etoposide inhibited VEGF secretion in U87 cells by $51 \%$ and in LLC cells by $36 \%$ (Fig. $1 \mathrm{H}$ ). The inhibi- tory effect of etoposide on VEGF secretion in vitro suggests a potential anti-angiogenic mechanism in vivo via decreased tumor cell production of this angiogenic mitogen.

Etoposide inhibits endothelial cell tube formation and FGF2- and VEGF-induced corneal neovascularization. To investigate whether etoposide has an effect on vessel morphogenesis, we seeded HUVECs on Matrigel, where they formed branching, anastomosing tubes that mimicked capillary-like structures (Fig. 2A). Etoposide inhibited tube formation in a dose-dependent manner (Fig. 2B-D), consistent with previous studies (23). To optimize the anti-angiogenic doses of etopo- 

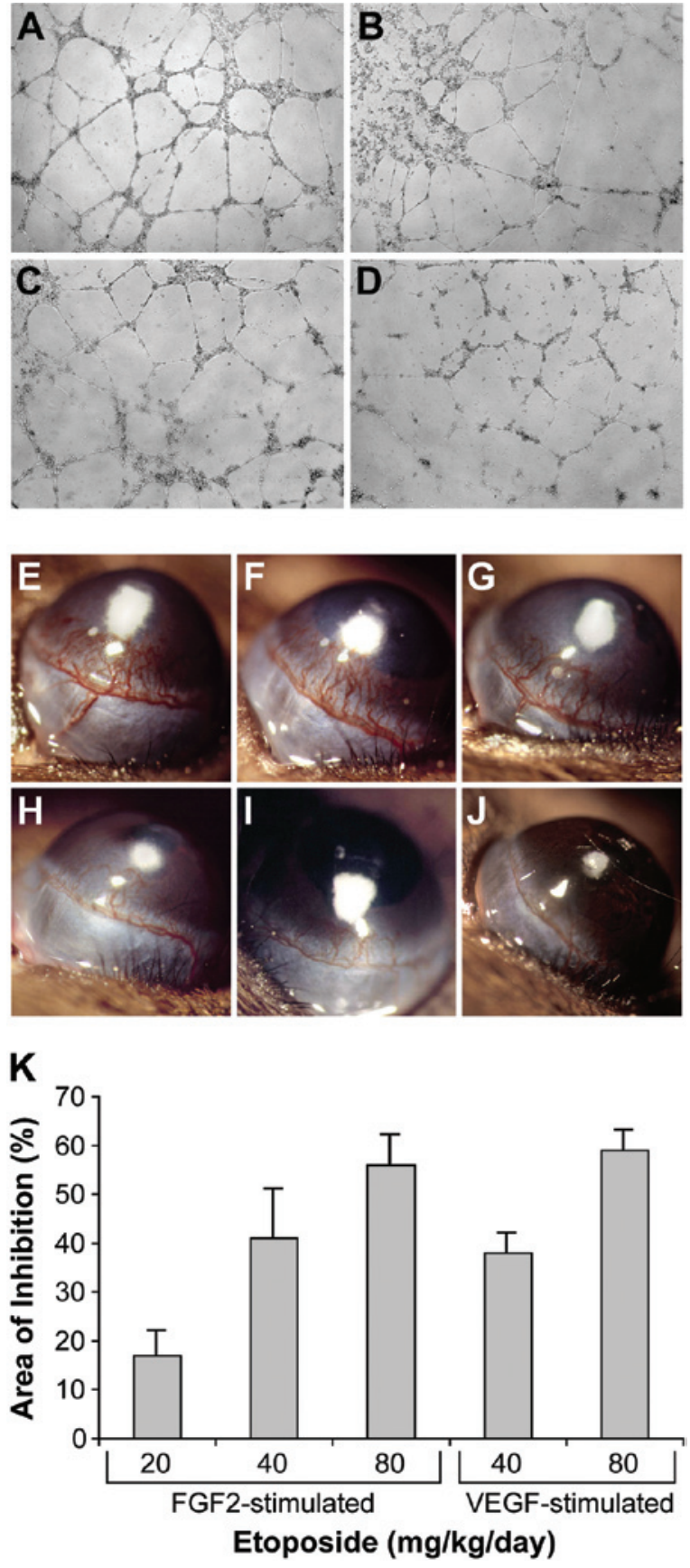

Figure 2. Etoposide inhibits endothelial cell tube formation (HUVEC morphogenesis on Matrigel) and FGF2- and VEGF-induced corneal neovascularization. (A) Representative photomicrograph of control tubes documenting the formation of a network of tube-like structures in HUVECs grown on Matrigel for $48 \mathrm{~h}$ in the presence of VEGF, FGF, HGF and IGF-1. (B-D) After a 48-h exposure to etoposide, dose-dependent inhibitory effects of etoposide were observed at (B) $25 \mu \mathrm{M}$, (C) $50 \mu \mathrm{M}$ and (D) $100 \mu \mathrm{M}$ (E) FGF2-induced corneal neovascularization in control cornea on Day 6. (F-H) Systemic oral treatment with etoposide at (F) $20 \mathrm{mg} / \mathrm{kg} / \mathrm{day},(\mathrm{G})$ $40 \mathrm{mg} / \mathrm{kg} /$ day and (H) $80 \mathrm{mg} / \mathrm{kg} / \mathrm{day}$. (I) VEGF-induced corneal neovascularization in control cornea on Day 5. (J) Systemic treatment with etoposide at $80 \mathrm{mg} / \mathrm{kg} /$ day. (K) Area of inhibition (\%) by various doses of daily etoposide. Inhibition was determined on Day 6 by the formula $0.2 \times \pi \times$ neovesse length $x$ clock hours of neovessels ( $n=5-6$ eyes/group; the experiment was performed three times).

side for daily administration in mice, we implanted $80 \mathrm{ng}$ FGF2 pellets into the corneas of C57BL/6 mice to stimulate neovascularization over 6 days (Fig. 2E). Systemic oral admin-
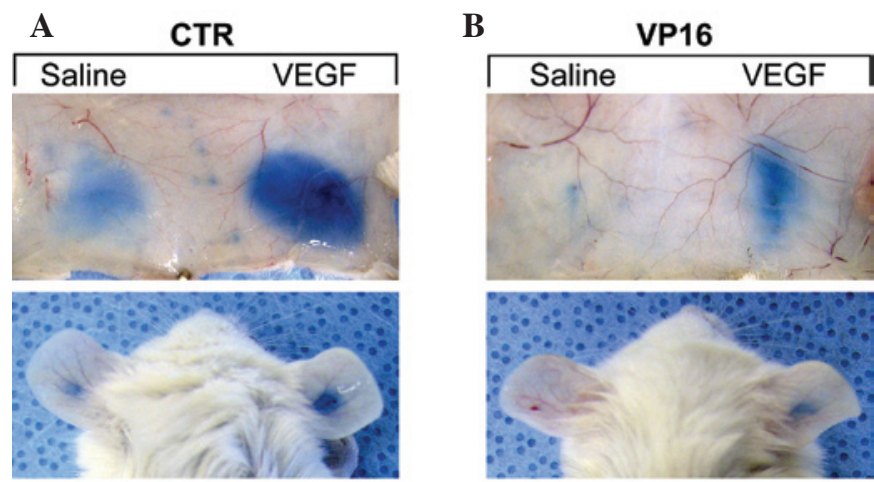

C
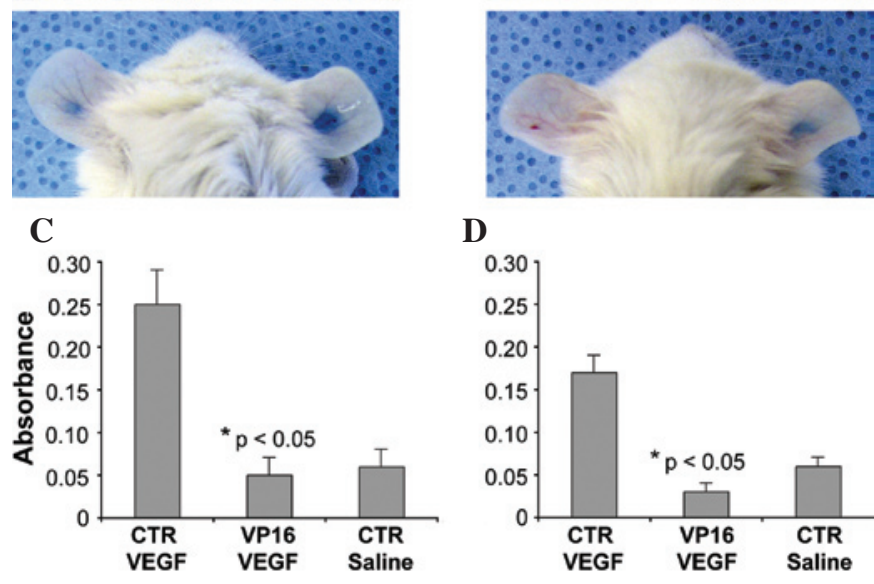

D
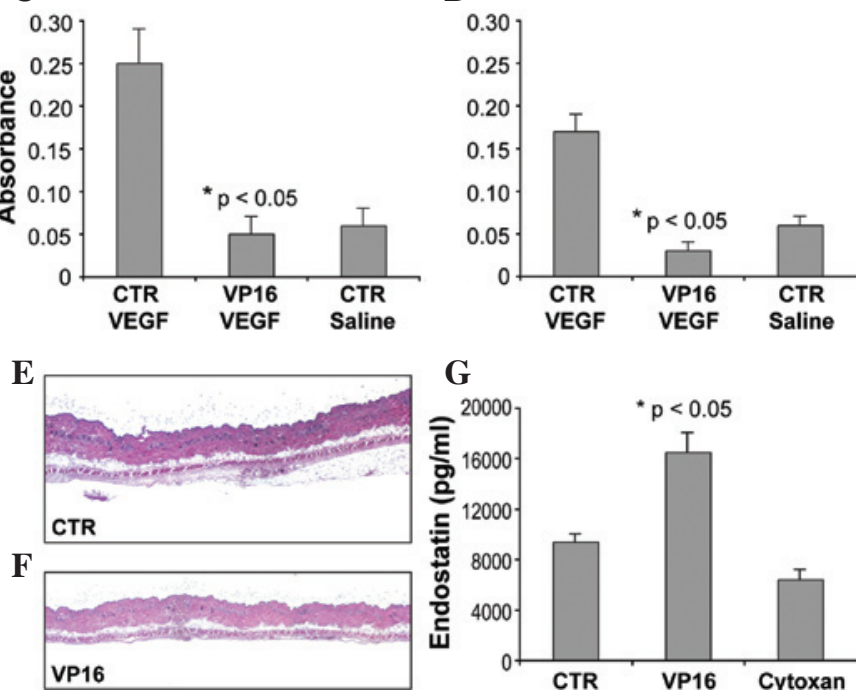

Figure 3. VEGF-induced vascular permeability is inhibited in mice treated with etoposide. (A and B) Evan's blue dye leakage in dorsal skin and ears after injection with VEGF or saline in control mice ( $\mathrm{n}=10 \mathrm{mice} / \mathrm{group}$ ) and etoposide (VP16)-treated mice ( $\mathrm{n}=10$ mice/group). (C and D) Spectrophotometric analysis of extravasated Evan's blue of the skin and ear is represented in bar graphs (average \pm standard deviation). (E and F) H\&E sections of skin from SCID mice treated with etoposide (VP16) or vehicle. (G) Etoposide, but not cyclophosphamide (cytoxan), elevated the endostatin level in vivo.

istration of etoposide significantly inhibited FGF2-induced corneal neovascularization in a dose-dependent fashion: $20 \mathrm{mg} / \mathrm{kg}$ /day resulted in 17\% inhibition (Fig. 2F); $40 \mathrm{mg} /$ $\mathrm{kg} /$ day resulted in $41 \%$ inhibition (Fig. $2 \mathrm{G}$ ); $80 \mathrm{mg} / \mathrm{kg} / \mathrm{day}$ resulted in 56\% inhibition (Fig. $2 \mathrm{H}$ ). To determine the effect of etoposide on VEGF-induced corneal neovascularization, VEGF pellets $(160 \mathrm{ng})$ were implanted into the corneas of C57BL/6 mice. Systemic oral administration of etoposide (40 and $80 \mathrm{mg} / \mathrm{kg} / \mathrm{day}$ ) inhibited VEGF-induced corneal neovascularization by 38 and 59\%, respectively (Fig. 2I and J). In summary, daily administration of etoposide exhibited dosedependent inhibition of both FGF2- and VEGF-stimulated corneal neovascularization (Fig. $2 \mathrm{~K}$ ).

Etoposide inhibits VEGF-induced vascular permeability and raises endostatin levels in vivo. We next determined whether etoposide (VP16) affects VEGF-induced vascular permeability, a standard test of in vivo VEGF activity (24). In response to VEGF, control mice displayed Evan's blue extravasation into the subcutaneous skin and ears (Fig. 3A) 80-82\% greater than that of etoposide-treated mice (Fig. 3B). 
A

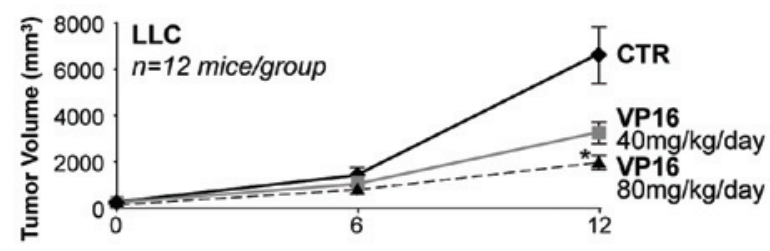

B

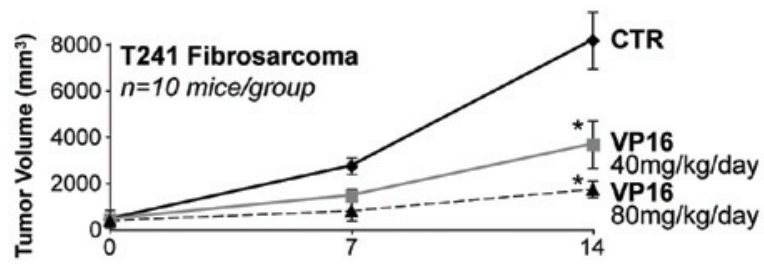

C

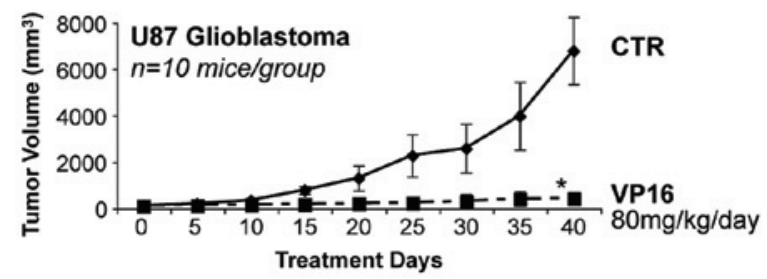

D
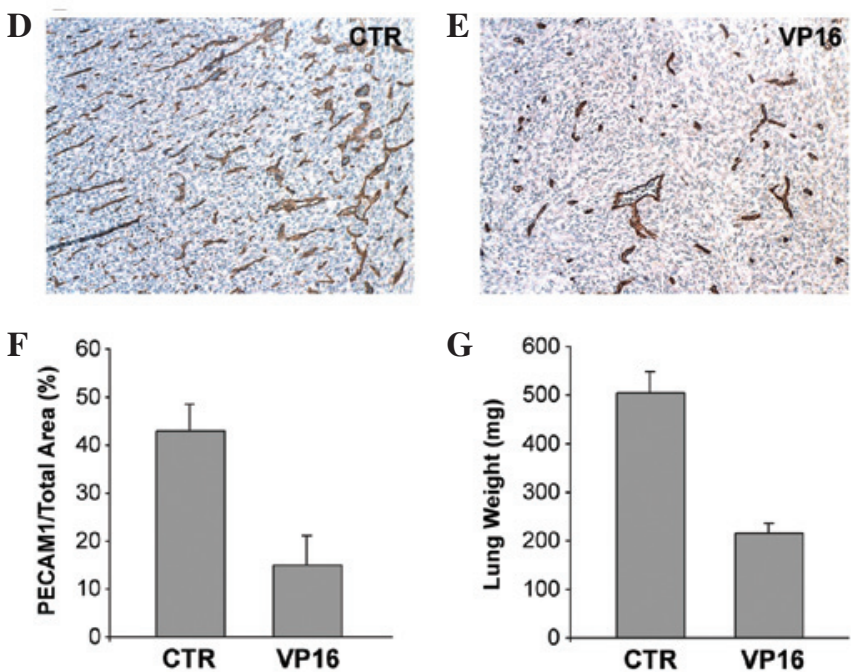

G

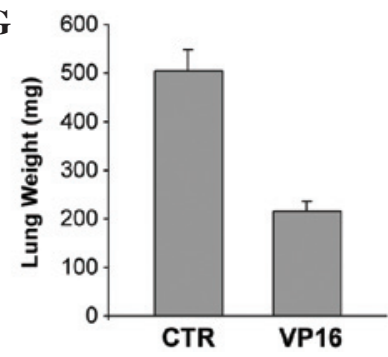

Figure 4. Systemic therapy with etoposide (VP16) inhibits primary tumor growth and metastasis by inhibiting angiogenesis. After the tumors reached $100-150 \mathrm{~mm}^{3}$ in size, etoposide treatment (40 or $80 \mathrm{mg} / \mathrm{kg} /$ day) was initiated (Day 0). On the last day of treatment, the statistical difference between the control and treated groups was determined by the Student's t-test. (A) LLC (P<0.05). (B) T241 fibrosarcoma $(\mathrm{P}<0.05)$. (C) Glioblastoma (U87) $(\mathrm{P}<0.001)$. (D) Representative immunostainings (PECAM1) of the vehicletreated and (E) etoposide-treated glioblastoma (U87) tumors. Brown color illustrates PECAM1-positive cells. (F) Vessel density in etoposide-treated Lewis lung carcinoma tumors as defined by the percentage of vessel area = PECAM1-positive area/tumor area in each field. (G) Etoposide inhibits lung metastasis, as represented by a significant decrease in lung weight, which correlates with metastatic tumor burden.

There was also a decrease in vascular leakage between the two saline groups in the etoposide-treated mice, presumably representing the inhibition of basal circulating VEGF. Spectrophotometric analysis of extravasated Evan's blue in both the skin and ear of etoposide-treated mice exhibited a dramatic reduction in VEGF-induced vascular permeability (Fig. 3C and D). Immunohistochemical analysis (H\&E staining) revealed that the area of skin edema was greatly
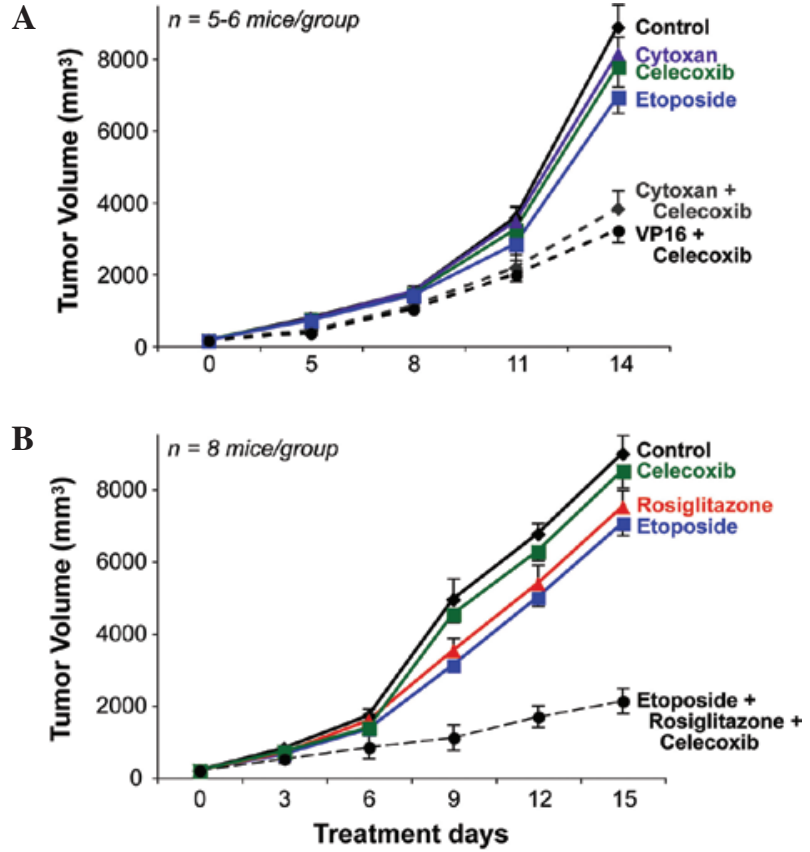

Figure 5. Etoposide (VP16) exhibits synergistic anti-tumor activity with oral anti-tumor and anti-angiogenic inhibitors. After the LLC tumors reached $100-150 \mathrm{~mm}^{3}$ in size, low-dose etoposide treatment $(10 \mathrm{mg} / \mathrm{kg} /$ day $)$ together with celecoxib (30 mg/ $\mathrm{kg} /$ day) or rosiglitazone $(50 \mathrm{mg} / \mathrm{kg} / \mathrm{day})$ was administered. Oral etoposide or sub-optimal doses of oral cyclophosphamide (cytoxan) $(10 \mathrm{mg} / \mathrm{kg} / \mathrm{day})$ were also administered in combination with (A) celecoxib or with (B) rosiglitazone and celecoxib (cyclophosphamide data not shown).

reduced in the etoposide-treated mice when compared to the vehicle-treated mice (Fig. 3E and F). Together, these results indicate that daily low-dose oral etoposide is a potent inhibitor of VEGF-dependent signaling.

Since etoposide raises biologically active endostatin levels in vitro (25), we examined whether the administration of etoposide raises endostatin levels in vivo. Mice treated with etoposide exhibited a $75 \%$ increase in plasma levels of endostatin (Fig. 3G). Another oral chemotherapeutic agent, cyclophosphamide, had no effect on endostatin levels in the plasma (Fig. 3G).

Systemic therapy with etoposide inhibits primary tumor growth and metastasis. In order to examine the antiangiogenic effect of daily, low-dose, oral etoposide (VP16) on the growth of primary tumors, we treated established subcutaneous tumors of $100-150 \mathrm{~mm}^{3}$ volume grown in mice. We utilized the optimal doses of etoposide identified in the corneal neovascularization assay. Oral etoposide at 40 and $80 \mathrm{mg} / \mathrm{kg} /$ day inhibited the growth of LLC by 29 and $56 \%$, respectively (Fig. 4A), and T241 fibrosarcoma by 55 and $79 \%$, respectively (Fig. 4B); $80 \mathrm{mg} / \mathrm{kg}$ of etoposide inhibited glioblastoma (U87) by $95 \%$ (Fig. 4C). There was no evidence of significant weight loss or other drug-related toxicity in any of the mice. To determine whether etoposide inhibited primary tumor growth by inhibiting angiogenesis, we measured the microvessel density in the treated and control tumors. A decrease in the microvessel density during treatment with an angiogenesis inhibitor suggests an anti-angiogenic effect on tumor growth (18). Etoposide treatment reduced microvessel 
density relative to that in the control tumors, thus indicating the presence of its anti-angiogenic efficacy (Fig. 4D-F).

Etoposide, when administered in mice via liposomes, was found to inhibit the formation of lung nodules in a metastatic tail vein model (26). The tail vein model illustrates only the homing step of tumor cells from circulation to an organ. By contrast, the LLC metastasis model is a model of spontaneous lung metastasis with all of the steps involved in metastasis including the invasion of tumor cells from the primary tumor to the circulation. Removal of the primary LLC was found to decrease the circulating angiogenesis inhibitor angiostatin, resulting in rapid growth of lung metastasis (21). In the present study, mice were treated for 15 days with oral daily etoposide $(80 \mathrm{mg} / \mathrm{kg} /$ day) or vehicle after removal of the primary LLC. In mice treated with vehicle, growing invasive metastasis almost entirely replaced the normal lung tissue, leading to lung weights in these mice of $505 \pm 42 \mathrm{mg}$. In marked contrast, mice treated with oral etoposide ( $80 \mathrm{mg} / \mathrm{kg} / \mathrm{day}$ ) had a lung weight of $216 \pm 19 \mathrm{mg}$ vs. normal lung weights of $152 \pm 10 \mathrm{mg}$ (Fig. 4G).

Etoposide has synergistic anti-tumor activity with oral antiangiogenic drugs, including celecoxib and rosiglitazone. To determine whether combining other classes of drugs improves the anti-tumor efficacy of etoposide, we utilized the cyclooxygenase-2 (COX2) inhibitor, celecoxib and peroxisome-proliferator activated receptor (PPAR) $\gamma$ ligand rosiglitazone, which are both orally administered and target endothelial and tumor cells $(22,27)$. We administered celecoxib, rosiglitazone and either etoposide or cyclophosphamide at the lowest doses necessary for minimal anti-tumor effect. Oral celecoxib (30 mg/kg/day) significantly enhanced the antitumor activity of low-dose oral etoposide (10 mg/kg/day) by $42 \%$ (Fig. 5A). When combined, PPAR $\gamma$ agonist rosiglitazone (50 mg/kg/day) and celecoxib (30 mg/ $/ \mathrm{kg} /$ day) enhanced the anti-tumor activity of low-dose oral etoposide $(10 \mathrm{mg} / \mathrm{kg} / \mathrm{day})$ by $69 \%$ (Fig. 5 B), with no evidence of drug-related toxicity.

\section{Discussion}

Most chemotherapy regimens are associated with significant toxicity when administered at maximum tolerated doses. There is now increasing evidence that multi-drug-resistant tumors are effectively targeted by anti-angiogenic chemotherapy $(1,2)$, in which low doses of cytotoxic drugs are given at close, regular intervals, with minimal toxic side effects (4). Therefore, standard chemotherapeutic agents, when modified by frequency and dose, target tumor angiogenesis. The mechanism by which cytotoxic chemotherapy affects the tumor vasculature may include selective killing of endothelial cells, suppression of circulating endothelial precursor cells and/or increasing levels of the endogenous angiogenesis inhibitors, such as thrombospondin-1 (4,28-31), and decreasing levels of angiogenesis stimulators, such as VEGF.

Oral etoposide, a chemotherapeutic drug, is an active agent in the treatment of various malignancies, including recurrent brain tumors, leukemia, lymphoma, hepatocellular carcinoma, Kaposi's sarcoma, ovarian and testicular cancer $(13,32-34)$. Patients with small-cell lung cancer treated with a prolonged maintenance of low serum etoposide concentrations $(>1$ $\mu \mathrm{g} / \mathrm{ml}$ ) were found to have a high response rate (35), while tumoricidal doses usually require $>10 \mu \mathrm{g} / \mathrm{ml}$ (36). Multiple pre-clinical and clinical studies have shown that the antitumor activity of etoposide is schedule-dependent, as smaller doses over several days or small daily doses result in higher response rates than single large doses $(12,14,32,37)$.

In addition to its effect on tumor cells, etoposide has been reported to reduce tumor angiogenesis in one of two renal cell carcinoma cell lines (38). Our studies support the role of etoposide in inhibiting angiogenesis in vitro and in vivo by decreasing VEGF production by tumor cells and microvessel density and increasing endostatin levels in vivo, consistent with other studies showing that etoposide increases the expression of biologically active endostatin in vitro (25). This increase in endostatin may explain in part the anti-tumor efficacy of etoposide (5). Results from our studies suggest that etoposide is an addition to the growing class of drugs that increase systemic endostatin levels, including tamoxifen, celecoxib and prednisolone plus salazosulphapyridine (in joint fluid) (39-41).

Tumor angiogenesis involves various pathways, thereby providing multiple molecular targets for anti-angiogenic drugs. Despite the potential efficacy of anti-angiogenic drugs, when used as single agents, resistance occurs by various mechanisms $(6,42)$. Therefore, there is an urgent need for multi-drug regimens in treating drug-resistant cancer in the clinic. Anti-angiogenic 'metronomic' chemotherapy with cyclophosphamide was shown to be synergistic with the thrombospondin peptide ABT-510 in suppressing tumor growth (43). Recent studies show synergy between PPAR $\gamma$ ligands, such as rosiglitazone, and platinum-based chemotherapeutic agents in inhibiting tumor growth (44). The use of oral etoposide in a number of combinations, such as with other angiogenesis inhibitors, chemotherapy and/or radiation, has demonstrated activity in mouse tumor models and patients $(19,25,45,46)$. Our results show that etoposide has synergistic anti-tumor activity with COX2 inhibitors and PPAR $\gamma$ ligands. COX2 inhibitors, such as celecoxib, have both anti-angiogenic and anti-tumor activities (27); we previously demonstrated that the PPAR $\gamma$ ligand rosiglitazone inhibits primary tumor growth and metastasis by targeting the tumor endothelium (22). The mechanism by which etoposide inhibits tumor angiogenesis may complement the anti-angiogenic effects of COX2 and PPAR $\gamma$ ligands resulting in greater inhibition of endothelial proliferation and a decrease in VEGF secretion.

Already, several human studies support the clinical relevance of oral etoposide. We recently incorporated etoposide as part of a four-drug anti-angiogenic chemotherapy regimen (thalidomide, celecoxib, etoposide and cyclophosphamide), which showed prolonged disease-free status in pediatric patients with recurrent or progressive cancer (7). Similarly, etoposide was part of a four-drug regimen named COMBAT (combined oral maintenance biodifferentiating and anti-angiogenic therapy), which was effective in solid tumors in children which had relapsed (9). This regimen included celecoxib, cis-retinoic acid, metronomic temozolomide and low-dose etoposide. Anti-angiogenic 'metronomic' chemotherapy is significantly cost-effective in the treatment of metastatic breast cancer (47). Therefore, oral etoposide, which is very well tolerated, may result in increased patient compliance; it can also be adminis- 
tered on an outpatient basis, thereby reducing costs, which is becoming an important issue $(48,49)$.

Our studies suggest that etoposide may be beneficial in treating angiogenic diseases, such as cancer, because of its effect on the endothelium and on angiogenesis pathways. Moreover, the endothelium is also an important target in the treatment of non-neoplastic diseases, such as arthritis, psoriasis and endometriosis. In fact, suboptimal doses of etoposide were found to improve collagen II-induced arthritis without monocyte depletion (50). As an orally administered FDA-approved drug, etoposide is ideally suited for use in combination with other anti-angiogenesis regimes and can complement conventional cancer treatment modalities.

\section{Acknowledgements}

We dedicate this research study to the memory of Dr Judah Folkman. The excellent technical assistance of Ricky Sanchez is acknowledged. We thank Kristin Johnson for photography. We thank Jessica Barnes for the helpful discussion. This study was supported by the Stop and Shop Family Pediatric Brain Tumor Fund and the C.J. Buckley Pediatric Brain Tumor Research Fund (M.W.K., D.P. and A.L.) and by the Department of Defense Innovator Award \#W81XWH-041-0316 (J.F.) and private philanthropic funds.

\section{References}

1. Browder T, Butterfield CE, Kraling BM, et al: Antiangiogenic scheduling of chemotherapy improves efficacy against experimental drug-resistant cancer. Cancer Res 60: 1878-1886, 2000.

2. Klement G, Baruchel S, Rak J, et al: Continuous low-dose therapy with vinblastine and VEGF receptor-2 antibody induces sustained tumor regression without overt toxicity. J Clin Invest 105: 15-24, 2000.

3. Hanahan D, Bergers $G$ and Bergsland E: Less is more, regularly: metronomic dosing of cytotoxic drugs can target tumor angiogenesis in mice. J Clin Invest 105: 1045-1047, 2000.

4. Kerbel RS and Kamen BA: The anti-angiogenic basis of metronomic chemotherapy. Nat Rev Cancer 4: 423-436, 2004.

5. Folkman J: Angiogenesis: an organizing principle for drug discovery? Nat Rev Drug Discov 6: 273-286, 2007.

6. Man S, Bocci G, Francia G, et al: Antitumor effects in mice of low-dose (metronomic) cyclophosphamide administered continuously through the drinking water. Cancer Res 62: 2731-2735, 2002 .

7. Kieran MW, Turner CD, Rubin J, et al: A feasibility trial of antiangiogenic (metronomic) chemotherapy in pediatric patients with recurrent or progressive cancer. J Pediatr Hematol Oncol 27: 573-581, 2005 .

8. Vogt T, Hafner C, Bross K, et al: Antiangiogenetic therapy with pioglitazone, rofecoxib, and metronomic trofosfamide in patients with advanced malignant vascular tumors. Cancer 98: 2251-2256, 2003.

9. Sterba J, Valik D, Mudry P, et al: Combined biodifferentiating and antiangiogenic oral metronomic therapy is feasible and effective in relapsed solid tumors in children: single-center pilot study. Onkologie 29: 308-313, 2006.

10. Aisner $\mathrm{J}$ and Lee EJ: Etoposide. Current and future status. Cancer 67: 215-219, 1991.

11. Kakolyris S, Samonis G, Koukourakis M, et al: Treatment of non-small cell lung cancer with prolonged oral etoposide. Am J Clin Oncol 21: 505-508, 1998.

12. Ashley DM, Meier L, Kerby T, et al: Response of recurrent medulloblastoma to low-dose oral etoposide. J Clin Oncol 14 1922-1927, 1996

13. Alici S, Saip P, Eralp Y, Aydiner A and Topuz E: Oral etoposide (VP16) in platinum-resistant epithelial ovarian cancer (EOC). Am J Clin Oncol 26: 358-362, 2003.
14. Martin M, Lluch A, Casado A, et al: Clinical activity of chronic oral etoposide in previously treated metastatic breast cancer. J Clin Oncol 12: 986-991, 1994.

15. Kebudi R, Gorgun O and Ayan I: Oral etoposide for recurrent/ progressive sarcomas of childhood. Pediatr Blood Cancer 42: 320-324, 2004

16. Cavalli F, Sonntag RW, Jungi F, Senn HJ and Brunner KW: VP-16-213 monotherapy for remission induction of small cell lung cancer: a randomized trial using three dosage schedules. Cancer Treat Rep 62: 473-475, 1978.

17. Bontenbal M, Planting AS, Verweij J, et al: Second-line chemotherapy with long-term low-dose oral etoposide in patients with advanced breast cancer. Breast Cancer Res Treat 34: 185-189, 1995.

18. Folkman J: Tumor angiogenesis. In: Cancer Medicine. Holland JF, Frei EI, Bast RCJ, Kufe DW, Pollock RE and Weichselbaum RR (eds). 5th edition. BC Decker Inc., Ontario, pp132-152, 2000.

19. Ma G, Masuzawa M, Hamada Y, et al: Treatment of murine angiosarcoma with etoposide, TNP-470 and prednisolone. J Dermatol Sci 24: 126-133, 2000.

20. Drevs J, Fakler J, Eisele S, et al: Antiangiogenic potency of various chemotherapeutic drugs for metronomic chemotherapy. Anticancer Res 24: 1759-1763, 2004.

21. O'Reilly MS, Holmgren L, Shing Y, et al: Angiostatin: a novel angiogenesis inhibitor that mediates the suppression of metastases by a Lewis lung carcinoma. Cell 79: 315-328, 1994.

22. Panigrahy D, Singer S, Shen LQ, et al: PPAR $\gamma$ ligands inhibit primary tumor growth and metastasis by inhibiting angiogenesis. J Clin Invest 110: 923-932, 2002.

23. Kamiyama H, Takano S, Tsuboi K and Matsumura A: Antiangiogenic effects of SN38 (active metabolite of irinotecan): inhibition of hypoxia-inducible factor 1 alpha (HIF-1alpha)/ vascular endothelial growth factor (VEGF) expression of glioma and growth of endothelial cells. J Cancer Res Clin Oncol 131: 205-213, 2005.

24. Dvorak HF: Vascular permeability factor/vascular endothelial growth factor: a critical cytokine in tumor angiogenesis and a potential target for diagnosis and therapy. J Clin Oncol 20: 4368-4380, 2002.

25. Hong SY, Lee MH, Kim KS, et al: Adeno-associated virus mediated endostatin gene therapy in combination with topoisomerase inhibitor effectively controls liver tumor in mouse model. World J Gastroenterol 10: 1191-1197, 2004.

26. Sant VP, Nagarsenker MS, Rao SG and Gude RP: Sterically stabilized etoposide liposomes: evaluation of antimetastatic activity and its potentiation by combination with sterically stabilized pentoxifylline liposomes in mice. Cancer Biother Radiopharm 18: 811-817, 2003.

27. Masferrer JL, Leahy KM, Koki A, et al: Antiangiogenic and antitumor activities of cyclooxgenase- 2 inhibitors. Cancer Res 60: 1306-1311, 2000.

28. Kerbel RS: Antiangiogenic therapy: a universal chemosensitization strategy for cancer? Science 312: 1171-1175, 2006.

29. Shaked Y, Emmenegger U, Francia G, et al: Low-dose metronomic combined with intermittent bolus-dose cyclophosphamide is an effective long-term chemotherapy treatment strategy. Cancer Res 65: 7045-7051, 2005

30. Bocci G, Nicolaou KC and Kerbel RS: Protracted low-dose effects on human endothelial cell proliferation and survival in vitro reveal a selective antiangiogenic window for various chemotherapeutic drugs. Cancer Res 62: 6938-6943, 2002.

31. Hamano Y, Sugimoto H, Soubasakos MA, et al: Thrombospondin-1 associated with tumor microenvironment contributes to low-dose cyclophosphamide-mediated endothelial cell apoptosis and tumor growth suppression. Cancer Res 64: 1570-1574, 2004

32. Needle MN, Molloy PT, Geyer JR, et al: Phase II study of daily oral etoposide in children with recurrent brain tumors and other solid tumors. Med Pediatr Oncol 29: 28-32, 1997.

33. Doll DC, Kasper LM, Taetle R and List AF: Treatment with low-dose oral etoposide in patients with myelodysplastic syndromes. Leuk Res 22: 7-12, 1998.

34. Hainsworth JD: Extended-schedule oral etoposide in selected neoplasms and overview of administration and scheduling issues. Drugs 58 (Suppl 3): 51-56, 1999.

35. Zucchetti M, Pagani O, Torri V, et al: Clinical pharmacology of chronic oral etoposide in patients with small cell and non-small cell lung cancer. Clin Cancer Res 1: 1517-1524, 1995. 
36. Lowis SP, Newell DR and Pearson AD: Exposure and schedule dependency of etoposide in neuroblastoma and leukaemia cells in vitro. Eur J Cancer 31A: 622-626, 1995.

37. Dombernowsky P and Nissen NI: Schedule dependency of the antileukemic activity of the podophyllotoxin-derivative VP 16-213 (NSC-141540) in L1210 leukemia. Acta Pathol Microbiol Scand [A] 81: 715-724, 1973.

38. Schirner M, Hoffmann J, Menrad A and Schneider MR: Antiangiogenic chemotherapeutic agents: characterization in comparison to their tumor growth inhibition in human renal cell carcinoma models. Clin Cancer Res 4: 1331-1336, 1998.

39. Nilsson UW and Dabrosin C: Estradiol and tamoxifen regulate endostatin generation via matrix metalloproteinase activity in breast cancer in vivo. Cancer Res 66: 4789-4794, 2006.

40. Ma L, del Soldato P and Wallace JL: Divergent effects of new cyclooxygenase inhibitors on gastric ulcer healing: shifting the angiogenic balance. Proc Natl Acad Sci USA 99: 13243-13247, 2002.

41. Nagashima M, Asano G and Yoshino S: Imbalance in production between vascular endothelial growth factor and endostatin in patients with rheumatoid arthritis. J Rheumatol 27: 2339-2342, 2000.

42. Yu JL, Rak JW, Coomber BL, Hicklin DJ and Kerbel RS: Effect of p53 status on tumor response to antiangiogenic therapy. Science 295: 1526-1528, 2002.

43. Yap R, Veliceasa D, Emmenegger U, et al: Metronomic low-dose chemotherapy boosts CD95-dependent antiangiogenic effect of the thrombospondin peptide ABT-510: a complementation antiangiogenic strategy. Clin Cancer Res 11: 6678-6685, 2005.
44. Girnun GD, Naseri E, Vafai SB, et al: Synergy between PPARgamma ligands and platinum-based drugs in cancer. Cancer Cell 11: 395-406, 2007.

45. Khafif A, Canfield VA, Syzek EJ and Medina JE: Results of phase I-II trial of concomitant hyperfractionated radiation and oral etoposide (VP-16) in patients with unresectable squamous cell carcinoma of the head and neck. Am J Otolaryngol 24: 1-5, 2003.

46. Vaishampayan U, Fontana J, Du W and Hussain M: Phase II trial of estramustine and etoposide in androgen-sensitive metastatic prostate carcinoma. Am J Clin Oncol 27: 550-554, 2004.

47. Bocci G, Tuccori M, Emmenegger U, et al: Cyclophosphamidemethotrexate 'metronomic' chemotherapy for the palliative treatment of metastatic breast cancer. A comparative pharmacoeconomic evaluation. Ann Oncol 16: 1243-1252, 2005.

48. Vanchieri C: When will the U.S. flinch at cancer drug prices? J Natl Cancer Inst 97: 624-626, 2005.

49. Emmenegger U, Morton GC, Francia G, et al: Low-dose metronomic daily cyclophosphamide and weekly tirapazamine: a well-tolerated combination regimen with enhanced efficacy that exploits tumor hypoxia. Cancer Res 66: 1664-1674, 2006.

50. Verdrengh $M$ and Tarkowski A: Impact of topoisomerase II inhibition on cytokine and chemokine production. Inflamm Res 52: 148-153, 2003. 